\title{
Noise Analysis for Strain Gauge Bridge Driving Circuit
}

\author{
Lihua Liu', a \\ ${ }^{1}$ School of Electrical and Information Engineering, Liaoning Institute of Science and Technology, \\ Benxi, China \\ a email: benxiliulihua@sina.com
}

Keywords: noise, weight scale, Strain Gauge Bridge, Resolution, Reference.

Abstract. The thesis calculates the noise of the strain gauge driving circuit in order to obtain high resolution. With the analysis the thesis points out the methods to reduce the noise. The thesis also draws the conclusion that an amplifier with lower noise spectral density but wide bandwidth perhaps is not suitable for the weigh scale driving circuit.

\section{Introduction}

Weight scale is widely used in the common life and commerce. It has very high resolution. The core part of it is the strain gauge bridge. Strain Gauge Bridge has some advantage: such as excellent linear character, lower temperature coefficient.

While bridge driving circuit produces very weak signal proportional to the measurement weight and it has most 0.01 to 0.02 volt when full scale. Most high resolution scales base on Strain Gauge Bridge.

In order to get high resolution, analog-to-digital converter (ADC) requires low noise input. The noise of driving circuit is a noise source, so we must estimate the noise and find out a way to reduce the noise for the high resolution. The thesis will introduce the method to calculate the noise and give out the method to choose the amplifier to reduce the noise to meet the strict requirement.

\section{Noise Value of Driving Circuit}

Assume the strain gauge bridge sensitivity is $2 \mathrm{mv} / \mathrm{V}$ and the bridge voltage is 5 volt and reference voltage is 2.5 volt. We want to acquire 10,000 resolutions.

The total input noise Vn of analog-to-digital converter must satisfy [1,2] :

$$
\frac{2.5}{6.6 V n}>10000
$$

From above equation, we get the range of Vn. It must satisfiy:

$$
V_{n}<38 u V
$$

From formula 2, we know the high resolution is so rigorous to the input noise.

Assume the PGA(programmable gain amplifier) is 128 and the noise of driving circuit voltage(bridge voltage) is Vnoise, then Vnoise should satisfy:

Vnoise $* 2 \mathrm{mv} / \mathrm{V} * 128<38 \mathrm{uV}$. 
From formula 3, we know Vnoise $<148 \mathrm{uV}$. If Vnoise is great then $148 \mathrm{uV}$, the resolution will be decreased. So we must estimate the true noise value.

Driving Circuit. In Fig.1, it is the typical driving circuit. U9 component is a high precision voltage reference, which is the input of the amplifier. The reference is typical 2.5 volt, so the output of amplifier is:

$$
V o=1+\frac{R 12}{R 11} \text {. }
$$

When R12=R11, the output voltage is 5 volt to drive the strain gauge bridge. The transistor CD9013, Q1 provides the abundant current to avoid the amplifier overheat. In the Fig.1, two capacity can filter the power supply noise to improve the accuracy.

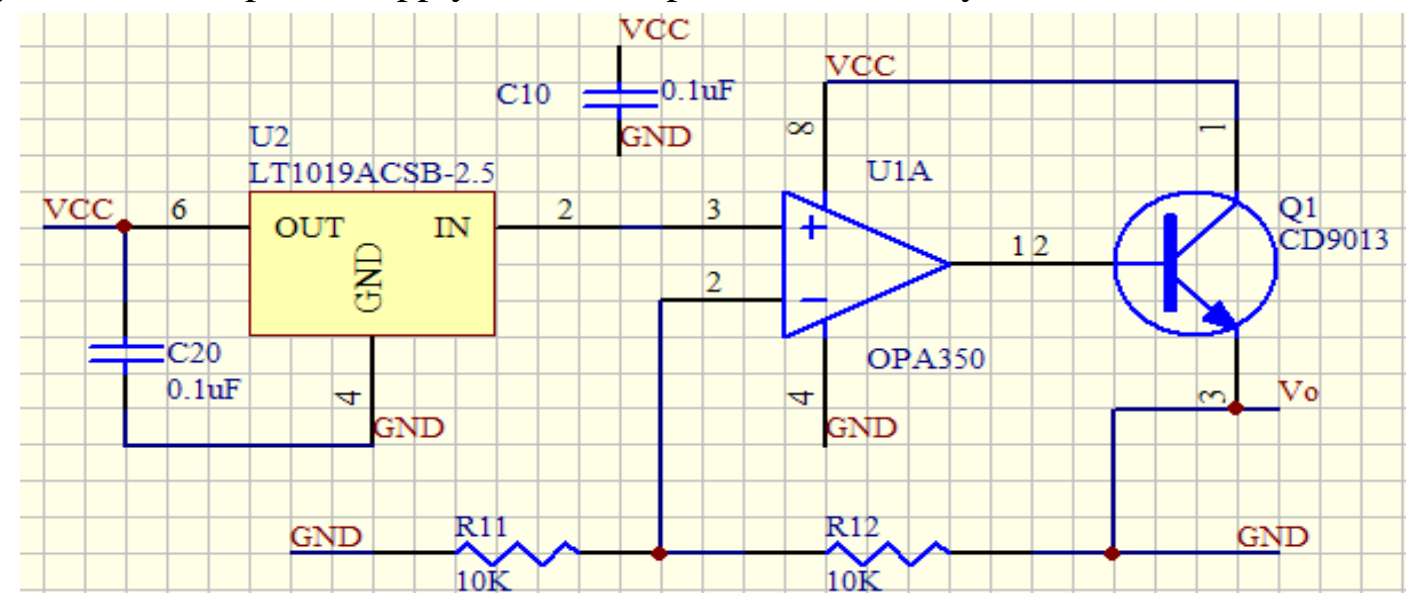

Fig .1. The typical driving circuit for the strain gauge bridge

Total Noise Analysis. The total input noise for analog-to-digital converter is consisted of four parts: reference voltage noise, resistor noise, amplifier voltage noise and current noise. The reference voltage noise is often so negligible, so we can consider the remains.

\section{Noise Analysis for Weigh Scale Driving Circuit}

Voltage noise includes two kinds [3]: white noise and 1/f noise.

$1 / \mathrm{f}$ noise lies in the lower frequency zone, it decrease while frequency increases. can it can calculate as following[4]:

$$
e n u_{-} f=e n o r m \sqrt{\ln \frac{f h}{f l}} \text {. }
$$

In above equation, enorm is the $1 / \mathrm{f}$ noise value at $1 \mathrm{~Hz}($ hertz); $f h$ is the upper frequency; $f l$ is lower cut frequency, typical we can consider it as $0.1 \mathrm{hertz}$ 。

White noise can calculate as following [4]:

$$
e n u_{-} b w=e u b b \sqrt{\Delta f} .
$$


In above equation, eubb is white noise effective value, $\Delta f$ is the white noise bandwidth。It is very plat in its bandwidth contra to the $1 / \mathrm{f}$ noise.

So the voltage noise can calculate as Eq. 5 .

$$
e n u=\sqrt{e n u_{-} f^{2}+e n u_{-} b w^{2}} \text {. }
$$

The total voltage noise is RTI (reference to input) .

Current noise is the same as voltage noise [4,5]:

$$
\begin{aligned}
& e n i_{-} f=R^{*} \text { Inorm } \sqrt{\ln \frac{f h}{f l}} . \\
& e n i_{-} b w=e i b b \sqrt{\Delta f} . \\
& e n i=\sqrt{e n i_{-} f^{2}+e n i_{-} b w^{2}} .
\end{aligned}
$$

In the formula 8 , it is the $1 / \mathrm{f}$ current noise. In the formula it is the current white noise. In the formula 10, it is the total current noise.

In the Fig.1, the resistance is the source of noise, too. Resistor noise can be calculated as following (RTI):

$$
e n r=\sqrt{4 * k^{*} T^{*} \operatorname{Re} q^{*} \Delta f}
$$

In formula $11, R e q=R_{11} / / R_{12}, T$ is Kelvin temperature degree, $k$ is the Boltzman constant. $\Delta f$ is the signal bandwidth. It can be determined by Equ.10 as following [4]:

$$
\Delta f=\frac{G B W}{A v} * 1.57
$$

In the formual 12 , symbol GWB is amplifier unit gain bandwidth; $A_{V}$ is amplifier magnification factor, in Fig. $1, A_{V}=2$.

So total peak-to-peak input noise can calculated [5]:

$$
e n=6.6 \sqrt{e n u^{2}+e n i^{2}+e n r e f^{2}+e n r^{2}} .
$$

\section{How to Reduce the Noise}

From Eq. 11, we know the value of equal resistor should be smaller, but too small value will cause more power consumption and cause the resistance increasing because of itself heat. So in the design, we take the R11,R12 as $10 \mathrm{Kohm}$.

From Eq. 6 ,Eq. 9 and Eq.11, amplifier bandwidth should be narrow.

From Eq.5 and Eq.8, we know the enorm should be smaller. 
From Eq.6 and Eq.9, we know current and voltage white noise spectral density should be smaller.

Above all, we know the amplifier characteristic decide the resolution.

In our typical concept, if an amplifier has very low enorm and white spectral density, then it own little noise. But we must notice sometimes it is not true. We omit a important factor, it is the $\Delta f$. If a wide amplifier takes the role as the weight scale driving circuit, it will case the serious noise though its price perhaps so expensive. If an amplifier has very limited bandwidth, while its enorm is not very small, the noise can be smaller than a wide bandwidth amplifier, though its price is perhaps is very cheap. Some FET(field effected transistor) input type amplifier often has nearly zero input current, the current 1/f noise can be omitted. So if a narrow bandwidth amplifier can achieve lower noise.

Sometimes we need to calculate the noise, it is trouble task for everyone. It is lucky that the amplifier manufacturer provide some software to calculate the noise to us for some kinds of amplifier, such TI_TINA, Linear pspice, and so on. WE can quickly know the actual noise from the amplifier output.

\section{The choose of Voltage Reference}

In some high resolution occasion, such as requiring the resolution is great than 10,000 , the noise of voltage reference must be accounted. This requiring the voltage has the very lower even lowest noise.

In the design, we choose LT1019 as the voltage reference; it has about $10 \mathrm{uV}$ noise from 0 to 10 $\mathrm{Hz}$ range. It is not very suitable for high resolution occasion; if possible, we can employee LT1021 as voltage reference. The noise from 0 to $10 \mathrm{~Hz}$ is only half of LT1019 [6].

\section{Conclusion}

In order to acquire the higher resolution for weight scale strain gauge bridge, we should choose the lower noise spectral density and low cutoff frequency amplifier. If possible, the FET amplifier with narrow bandwidth can achieve the lower noise as expensive amplifier mentioned.

The resistor, which magnifies the voltage reference, should be small. But the value must be over $1 \mathrm{Kohm}$ as the balanced point between the accuracy and noise.

But opposite common thinking, the product of noise spectral density and bandwidth decides the resolution while not the noise spectral density.

In some higher solution occasion, the voltage reference must be selected carefully. It can also output noise, which will decrease the resolution. In the design, recommend the LT1021 replace the LT1019 because of its very low noise.

\section{References}

[1] Information on http://www.ti.com

[2] Information on http://www.silabs.com

[3] Hui Tian; Fowler, B.; Gamal, A.E.: Solid-State Circuits, IEEE Journal of, Vol.36(2001), p.92

[4] Dai, Y.: Circuits, Devices and Systems, IEE Proceedings, Vol.145(1998),p.343 
[5] Won Ko; Youngwoo Kwon: Microwave Theory and Techniques, IEEE Transactions on, Vol.53 (2005), p.361

[6] Information on http://www.linear.com 